\title{
Possibility of observing energy decoherence due to quantum gravity
}

\author{
Christoph Simon ${ }^{1}$ and Dieter Jaksch ${ }^{2}$ \\ ${ }^{1}$ Laboratoire de Spectrométrie Physique, CNRS et Université Joseph Fourier Grenoble-I, F-38402 St. Martin d'Hères, France \\ ${ }^{2}$ Clarendon Laboratory, University of Oxford, Parks Road, Oxford OX1 3PU, United Kingdom
}

(Received 1 June 2004; published 10 November 2004)

\begin{abstract}
It has recently been proposed that quantum gravity might lead to the decoherence of superpositions in energy, corresponding to a discretization of time at the Planck scale. The proposal seems amenable to experimental verification with methods from quantum optics and atomic physics. However, we argue that the predicted decoherence is unobservable in such experiments if it acts globally on the whole experimental setup. This is related to the unobservability of the global phase in interference. We then show how local energy decoherence, which acts separately on system and phase reference, could be detected with remarkable sensitivity and over a wide range of length scales by long-distance Ramsey interferometry with metastable atomic states. The sensitivity of the experiments can be further enhanced using multiatom entanglement.
\end{abstract}

DOI: 10.1103/PhysRevA.70.052104

PACS number(s): 03.65.Yz, 04.60.-m, 42.50.Xa, 03.75.Gg

\section{INTRODUCTION}

The unification of quantum physics and gravitation is one of the big open questions in physics. A large amount of theoretical work is devoted to it, following various approaches, including string theory, loop quantum gravity and others. Experimental guidance would be extremely valuable. Some predictions of string theory, such as supersymmetry, should be testable in future high-energy particle accelerators. Certain candidate theories predict deviations from the usual dispersion relations for very energetic particles. Astrophysical observations can give constraints on such predictions [1].

Another class of theoretical predictions from quantum gravity concerns the decoherence of quantum superpositions $[2,3]$. In particular it has recently been predicted based on discrete quantum gravity that there should be decoherence in the energy basis [4]. Such energy decoherence can be understood intuitively as arising from a discretization of time at a very small scale [5]. Even though there is no universal agreement that quantum gravity implies energy decoherence, it seems to us that the proposal deserves serious attention, not only because it is related to a very basic concept (the discretization of time), but also because it might lend itself to experimental verification with the methods of quantum optics and atomic physics, i.e., essentially tabletop experiments [4].

Reference [4] predicts that the evolution equation for the density matrix $\rho$ of a quantum system with Hamiltonian $H$ should be given by

$$
\frac{d \rho}{d t}=\frac{i}{\hbar}[H, \rho]-\frac{\sigma}{\hbar^{2}}[H,[H, \rho]],
$$

where the constant parameter $\sigma$, which is essentially the discretization time scale, may be as small as the Planck time $t_{P}$, i.e., of order $10^{-43} \mathrm{~s}$. This time evolution leads to a decay of off-diagonal terms in the energy basis $|E\rangle\langle E+\Delta E|$ with a rate $\gamma_{Q G}$ of order $\sigma(\Delta E / \hbar)^{2}$.

In this paper we address the question whether this type of decoherence could be observed in practice. We believe that the only presently conceivable type of experiment that has the potential to yield nontrivial bounds on the parameter $\sigma$ of Eq. (1) is to prepare a superposition of two states with substantially different energies and observe its decoherence, trying to separate environmental and potentially present quantum gravitational effects. The quadratic dependence on $\Delta E$ of the decay rate for such superpositions is a decisive advantage compared to effects for wavepackets with a smooth distribution in energy, which would only become significant for energies close to the Planck energy, cf. Ref. [5].

The most promising approach to achieve such superpositions is the use of long-lived metastable atomic states that are separated from the ground state by optical transitions, such as the ${ }^{3} P_{2}$ and ${ }^{3} P_{0}$ states in strontium [6]. A superposition $|g\rangle+|e\rangle$ between the ground state and such an excited state has a $\Delta E$ of order $1 \mathrm{eV}$. Note that this is about seven orders of magnitude larger than, for example, the cavity-induced energy splitting discussed in [4] would be in a realistic experiment [7]. Moreover, because the spontaneous lifetime of the excited state can be of the order of hundreds of seconds [6], an experiment with a single atom could in principle be sensitive to a decoherence rate at the level of $10^{-3} / \mathrm{s}$. This can again be compared for illustration to the example discussed in Ref. [4], where typical cavity lifetimes are of order microseconds [7]. Such sensitivity to decoherence rates $\gamma_{Q G}$ in the $\mathrm{mHz}$ regime would allow one to detect $\sigma$ at the level of $10^{-33} \mathrm{~s}$. This remarkable sensitivity could be further improved by several orders of magnitude using multiatom entangled states of the GHZ type, $|e\rangle^{\otimes N}+|g\rangle^{\otimes N}$. We will discuss the possibility of using multiatom entanglement in more detail below.

However, we first have to point out a fundamental problem concerning the observability of global energy decoherence in the quantum optical domain. Coherence in energy (and therefore also decoherence) is in practice observed interferometrically by studying the phase of the quantum system under consideration relative to a given phase reference. Only the relative phase between system and reference is observable. At the fundamental level the phase reference also has to be treated as a quantum system. Energy decoherence that acts globally on system and reference together only has an effect on the global phase of the combined system, but 
does not influence the relative phase between the two parts. It is therefore unobservable. We discuss this rather subtle point in detail in Sec. II with the help of illustrative examples. This part of our work can be seen as an extension of the arguments by Finkelstein [8] to realistic experimental situations. We conclude this section by arguing that the need for a phase reference, and thus the unobservability of global decoherence, fundamentally arises from the fact that macroscopic systems have very little coherence in energy.

In contrast to global decoherence, local energy decoherence that acts separately on the system and the reference can be observable. In Sec. III we describe several experimental approaches that might allow to test energy decoherence which is local on various length scales, ranging from micrometers to possibly millions of kilometers. Atom and molecule interferometry already give Planck-level bounds on $\sigma$ for decoherence that is local on $\mu \mathrm{m}$ scales. Ramsey interferometry with a large separation between the laser and the atom is very promising for detecting discretization timescales $\sigma$ down to $10^{-33} \mathrm{~s}$, over length scales up to the order of kilometers for terrestrial experiments. The use of multiatom entangled states, whose creation should be possible in BoseEinstein condensates in the foreseeable future, could enhance the sensitivity down to the level of $10^{-38} \mathrm{~s}$. It might be possible to go beyond terrestrial length scales by many orders of magnitude in space-based experiments using projected technology. Given the large theoretical uncertainties for possible values of $\sigma$ in gravitational decoherence [4] and the ability of the proposed experimental setups to test a number of alternative decoherence models $[9,10]$ it is certainly worthwhile to further improve the sensitivity of interference experiments, not least to obtain some guidance for future theoretical studies.

\section{UNOBSERVABILITY OF GLOBAL ENERGY DECOHERENCE}

\section{A. Ramsey interference}

We will first illustrate the problem by discussing a single atom that can be in states $|g\rangle$ or $|e\rangle$, but we will argue below that it is much more general. It is important to consider how a superposition of the type $|g\rangle+|e\rangle$ would in practice be created and observed, namely by applying laser pulses that induce Rabi rotations between $|g\rangle$ and $|e\rangle$. The experimental technique is known as Ramsey interferometry. The first pulse creates the superposition $|g\rangle+|e\rangle$ from the initial state $|g\rangle$, followed by a variable delay, during which the two terms in the superposition acquire a relative phase, such that the state evolves to $|g\rangle+e^{i \phi}|e\rangle$, and during which decoherence can act. The second pulse is such that it brings the system back to $|g\rangle$ with unit amplitude if the relative phase $\phi$ is zero and if no decoherence has occurred. If $\phi$ is different from zero, the probability to observe the system in $|g\rangle$ will be different from one, leading to Ramsey interference fringes in dependence on the phase. Energy decoherence as described by Eq. (1) will affect the visibility of these fringes. In particular, for complete decoherence the superposition is transformed into an equal mixture $\frac{1}{2}(|g\rangle\langle g|+| e\rangle\langle e|)$, in which all phase information is lost and which is unchanged by the second laser pulse. Note that at the end the system is always detected in either $|e\rangle$ or $|g\rangle$, i.e., the detection is performed in the basis spanned by the energy eigenstates, not in a basis of superposition states. This detection is typically performed by detecting fluorescence (under laser excitation) from a third state that is accessible only from one of the two states.

Ramsey interference occurs because there are two different histories that can lead to the same final state. Suppose that at the end the atom is detected in $|e\rangle$. Then it can have absorbed a photon from the first pulse and acquired a phase $\phi$ during the intermediate waiting time, or it can have absorbed a photon from the second pulse and thus not acquired a phase. The amplitudes for the two histories have to be added. The two histories are distinguished by the energy of the atom during the waiting time. It therefore seems at first sight that the experiment should be sensitive to decoherence in the energy basis.

Conventionally in the description of Ramsey interference the laser light is treated as a classical system, which is usually an excellent approximation. However, for our present purpose it is essential to take the quantum character of the light into consideration. The Hamiltonian for the interaction between the atom and the light is

$$
H=g\left(a|e\rangle\left\langle g\left|+a^{\dagger}\right| g\right\rangle\langle e|\right) .
$$

This is in the rotating wave approximation, which is extremely well justified in the relevant regime where the pulse durations are much longer than an optical period. This Hamiltonian describes the exchange of excitations between the light and the atom. The destruction of a photon is accompanied by the creation of an atomic excitation and vice versa. The total energy of the combined system light plus atom is not changed by the action of this interaction Hamiltonian.

The Ramsey interference can be analyzed independently in subspaces of fixed total energy, that is for initial states $|N\rangle|g\rangle$, where $N$ is the total number of photons. Let us first consider an idealized situation where the same laser pulse is made to interact twice with the atom. The first interaction creates a superposition of the two terms $|N\rangle|g\rangle$ and $\mid N$ $-1\rangle|e\rangle$. Both terms can give rise to the states $|N\rangle|g\rangle$ and $\mid N$ $-1\rangle|e\rangle$ after the second interaction. The relative phase that the two terms acquire between the interactions therefore determines the observable interference effects (the probabilities for the atom to be in $|g\rangle$ or $|e\rangle)$. Obviously the two terms have the same total energy. The observable effects are therefore completely independent of the presence of global energy decoherence, that is of energy decoherence that acts on the light and the atom as on a single system.

It is always possible to analyze the interference in this way, even if the light is not initially prepared in a Fock state $|N\rangle$. In particular, this is also true for the case where the state of the light is a macroscopic coherent state, such that it remains essentially unchanged by the exchange of photons with the atom, and always factorized from the atomic state. Our statement here is not that a true superposition state of the atom can never be created, which is true for an initial Fock state of the light field. The essential point is that in any case only the interference within each pair of histories with the same total energy matters for the observable effects. 
The same conclusion can be reached in a more realistic situation, where the light is split into two pulses, that interact sequentially with the same atom. Again the analysis can be performed in subspaces of fixed total energy. The initial $N$ photon state is split coherently into two parts, corresponding to the first and the second pulse. This creates a state of the form $\Sigma_{n} c_{n}|N-n\rangle_{1}|n\rangle_{2}|g\rangle$. The first pulse interacts with the atom, such that every component $|N-n\rangle_{1}|g\rangle$ is transformed into a superposition $a_{n}|N-n\rangle_{1}|g\rangle+b_{n}|N-n-1\rangle_{1}|e\rangle$, where the interaction is adjusted such that the coefficients $a_{n}$ and $b_{n}$ are equal to $1 / \sqrt{2}$ with good approximation. After this interaction the first pulse never comes back. Therefore it does not contribute to the interference. The global state after the interaction between the first pulse and the atom can be rewritten as

$$
\sum_{n}|N-n\rangle_{1}\left(c_{n} a_{n}|n\rangle_{2}|g\rangle+c_{n-1} b_{n-1}|n-1\rangle_{2}|e\rangle\right) .
$$

Tracing over the first pulse, one sees that the relevant interference is between pairs of terms of the form $|n\rangle_{2}|g\rangle$ and $\mid n$ $-1\rangle_{2}|e\rangle$, that is between pairs of states with the same energy. Both of these terms can lead to final states $|n\rangle_{2}|g\rangle$ and $\mid n$ $-1\rangle_{2}|e\rangle$ after the interaction between the second pulse and the atom. Therefore the phase between them determines the final probability for the atom to be detected in $|g\rangle$ or $|e\rangle$. Again the relevant interference is between states with the same total energy, and the final probability for the atom to be in $|g\rangle$ or $|e\rangle$ is completely independent of energy decoherence that acts on the second pulse and the atom as on a single system.

This unobservability of global energy decoherence is not specific to Ramsey interference. We argue that it is universal, at least for the domain of quantum optics. The basic reason is that in every experiment conceivable to us in the quantum optical regime the final detection is performed in the energy basis. Superpositions in energy, such as the fixed phase relationship between $|g\rangle$ and $|e\rangle$, are detected with the help of a phase reference. In Ramsey interference the superposition is created by the first laser pulse, while the second laser pulse serves as the phase reference. What matters for the experimental results is the relative phase between system and the reference. The global phase is not observable. However, it is only this global phase that is affected by global energy decoherence.

\section{B. Michelson interference}

To further emphasize and clarify this important point, consider a very simple example of such an interference experiment, a Michelson interferometer for light. We denote the two input modes of the interferometer by $a$ and $b$, and the modes traveling towards the mirrors in the two arms by $c$ and $d$. We start with a coherent state $|\alpha\rangle$ in mode $a$ and the vacuum in mode $b$, i.e., an initial state

$$
|\alpha\rangle_{a}|0\rangle_{b}=e^{-|\alpha|^{2} / 2} \sum_{n=0}^{\infty} \frac{\alpha^{n}}{\sqrt{n !}}|n\rangle_{a}|0\rangle_{b}=e^{-|\alpha|^{2} / 2} e^{\alpha a^{\dagger}}|0\rangle,
$$

where $|0\rangle$ is the vacuum of all modes. The modes $c$ and $d$ are related to the inputs by $a=(c+d) / \sqrt{2}$ and $b=(c-d) / \sqrt{2}$, such that

$$
|\alpha\rangle_{a}|0\rangle_{b}=\left|\frac{\alpha}{\sqrt{2}}\right\rangle_{c}\left|\frac{\alpha}{\sqrt{2}}\right\rangle_{d}
$$

The incoming light is split equally between the two arms. The modes $c$ and $d$ are transformed into modes $\tilde{c}$ and $\tilde{d}$ respectively, which travel from the mirrors back to the beamsplitter. For a perfectly balanced interferometer the output modes $\tilde{a}$ and $\tilde{b}$ are related to $\tilde{c}$ and $\tilde{d}$ in the same way as $a$ and $b$ are to $c$ and $d$. In particular one therefore has $a=(c$ $+d) / \sqrt{2}=(\tilde{c}+\tilde{d}) / \sqrt{2}=\tilde{a}$.

The coherent states propagating in the two arms are coherent superpositions of states of different photon numbers and thus different energies. It might therefore seem that global energy decoherence should have an effect on the interference, such that in the presence of decoherence some photons would end up in mode $\tilde{b}$ in the final state. However, the effect of complete global energy decoherence is to transform the state $|\alpha\rangle_{a}|0\rangle_{b}$ into a Poissonian mixture of Fock states,

$$
\rho=\sum_{n=0}^{\infty} e^{-|\alpha|^{2}} \frac{|\alpha|^{2 n}}{n !}|n\rangle_{a}\langle n \| 0\rangle_{b}\langle 0| .
$$

This input state is exactly reproduced in the output modes, because the interference happens independently for every total photon number, according to

$$
\left(a^{\dagger}\right)^{n}|0\rangle=\left(\frac{c^{\dagger}+d^{\dagger}}{\sqrt{2}}\right)^{n}|0\rangle=\left(\frac{\widetilde{c}^{\dagger}+\tilde{d}^{\dagger}}{\sqrt{2}}\right)^{n}|0\rangle=\left(\widetilde{a}^{\dagger}\right)^{n}|0\rangle .
$$

The coherence (or its absence) between different total photon numbers is irrelevant for the Michelson interference. This is in full analogy to Ramsey interference, which, as we have seen above, can also be analyzed separately for every total photon number. Equivalently, the global phase of the initial state $|\alpha\rangle$ is irrelevant, only the relative phase between the states in the two arms is important. This relative phase remains unaffected by the global decoherence. This can also be seen by noting that the decohered state can be written as

$$
\rho=\frac{1}{2 \pi} \int_{0}^{2 \pi} d \phi\left|\alpha e^{i \phi}\right\rangle_{a}\left\langle\alpha e^{i \phi}|| 0\right\rangle_{b}\langle 0|,
$$

which in terms of the modes $c$ and $d$ is

$$
\frac{1}{2 \pi} \int_{0}^{2 \pi} d \phi\left|\frac{\alpha}{\sqrt{2}} e^{i \phi}\right\rangle_{c}\left\langle\frac{\alpha}{\sqrt{2}} e^{i \phi}|| \frac{\alpha}{\sqrt{2}} e^{i \phi}\right\rangle_{d}\left\langle\frac{\alpha}{\sqrt{2}} e^{i \phi}\right| .
$$

This shows that the relative phase between the two modes is unaffected, even though the reduced density matrix of each individual mode ( $c$ and $d$ ) after global energy decoherence is given by

$$
\frac{1}{2 \pi} \int_{0}^{2 \pi} d \phi\left|\frac{\alpha}{\sqrt{2}} e^{i \phi}\right\rangle\left\langle\frac{\alpha}{\sqrt{2}} e^{i \phi}\left|=\sum_{n=0}^{\infty} e^{-|\alpha|^{2} / 2} \frac{|\alpha|^{2 n}}{2^{n} n !}\right| n\right\rangle\langle n|,
$$

which is a Poissonian mixture of Fock states without any phase relation. 


\section{Time domain experiments}

We claim that these simple examples are in fact generic. In particular, the arguments apply to Ramsey type experiments that use GHZ states of the form $|g\rangle^{\otimes N}+|e\rangle^{\otimes N}$ instead of single atoms. They also apply to experiments that would aim to demonstrate coherence and decoherence in the energy basis via time measurements. For example, one could argue that the shortness in time of a light pulse from a mode-locked laser demonstrates coherence in energy and that decoherence should lead to an observable broadening of the pulse in time. However, what is really measured in practice is the relative time between the pulse and a reference pulse. In the simplest case, the original pulse is split into two parts, which are recombined in a nonlinear medium, where two coincident photons (one from each pulse) can combine to give a single photon of higher energy, which is detected. All such experiments are sensitive only to the relative time between system and reference, in analogy to the relative phase for the above experiments, and thus only allow inference about the coherence in the energy difference between system and reference, but not about coherence in the total energy. Again, the relevant interference occurs between states of fixed total energy, which is shared in different ways between system and reference. This point was made previously in a slightly different language, but also in the time domain, by Finkelstein [8]. The present point of view is also a good way of understanding the results of the Gedanken experiments discussed very recently by Pearle [11] in the context of energy-driven collapse models [9].

\section{Discussion}

Global energy coherence (and thus decoherence) is unobservable in the above experiments because the coherence is observed with respect to a phase reference. As long as this phase reference has to be treated as part of the quantum system that is subject to energy decoherence, the decoherence is unobservable. If on the other hand the decoherence acts separately on the system and the phase reference, it can have observable effects. We will pursue this possibility in the following section. Another theoretical possibility allowing observation of the decoherence would be for the phase reference to remain unaffected by the decoherence, which would only act on the system. This would correspond to the case of a "completely classical" phase reference, which could only exist if there were somewhere a border between the quantum and the classical world.

But what are the reasons underlying the need for a phase reference? In a certain sense this requirement is of a practical, not fundamental, nature. One could detect global energy decoherence without a phase reference, if there was a macroscopic physical system which could be in long-lived states with large $\Delta E$ that are macroscopically distinct from states without significant energy coherence. In this case energy decoherence would be directly observable. However, we suspect that no such physical system can exist in practice. Macroscopic systems are always in contact with their environment (usually even in thermal equilibrium). As a consequence, the states in which they are observed have very little coherence in the energy basis. In particular, this applies to the macroscopic systems used to indicate measurement results in typical experiments. For instance, in the cases considered above the measurements are done by counting photons (from fluorescence in the case of the Ramsey experiment, from up-conversion in the case of the short pulses, directly from the laser in the Michelson experiment). In these setups only energetically distinct states (absence or presence of a photon) lend themselves to amplification to the macroscopic level.

It is worth pointing out that the absence of energy coherence with large $\Delta E$ for individual systems would in fact be implied by the presence of global energy decoherence. With the age of the universe of order $10^{10}$ years, one finds that even for $\sigma$ from Eq. (1) of order the Planck time $t_{P}$ all coherences with $\Delta E$ larger than a few meV would have decayed. Global energy decoherence acting on the whole universe would thus have essentially reduced it to a mixture of energy eigenstates at the present moment. Note that this would not necessarily have any observable consequences for experiments using phase references or other clocks, for the reasons discussed in this section. In a universal energy eigenstate $\left|E_{u}\right\rangle$, every energy state $|E\rangle$ of an isolated subsystem is correlated with a state $\left|E_{u}-E\right\rangle$ for the rest of the universe, so that no coherence can exist in the individual system. Global energy decoherence could thus have itself destroyed the conditions for its observation.

\section{TESTING LOCAL ENERGY DECOHERENCE}

In the previous section we have arrived at the conclusion that the prediction of Ref. [4] is likely to be untestable if the decoherence is assumed to act globally. It is therefore important to understand whether there is a length scale on which the decoherence might act locally. The question of the spatial dependence of the energy decoherence was already raised by Milburn in Ref. [5], based on considerations of Lorentz invariance. We make no attempt to answer this question here. However, we outline some experimental approaches that should allow to test energy decoherence that is local on various length scales.

\section{A. Atom and molecule interferometry}

Atom and molecule interferometry are extremely sensitive to the occurrence of energy decoherence that acts locally on short length scales. For example, separations of up to $20 \mu \mathrm{m}$ between the two paths were achieved in an interferometry experiment with sodium atoms [12]. The rest mass of a sodium atom corresponds to an energy of order $20 \mathrm{GeV}$. Even for $\sigma$ in Eq. (1) of order the Planck time, this implies a decoherence rate of order $10^{8} / \mathrm{s}$, if the decoherence acts separately on each path. For an atom velocity of $3000 \mathrm{~m} / \mathrm{s}$ as in Ref. [12], this would imply that the atoms should be significantly decohered after propagating just $30 \mu \mathrm{m}$. The calculated decoherence rates would be even more dramatic in molecule interferometry experiments such as Ref. [13], however the achieved path separations are only of order $1 \mu \mathrm{m}$. Energy decoherence acting locally below the $\mu \mathrm{m}$ length 
scale is thus already ruled out by these experiments. A further improvement of energy decoherence bounds on short length scales could be achieved by utilizing multiparticle entanglement enhanced atom interferometers [14].

\section{B. Long-distance Ramsey interferometry}

As discussed above, the relevant interference in a Ramsey experiment is between states of the form $|g\rangle|n\rangle$ and $|e\rangle \mid n$ $-1\rangle$, where $|g\rangle$ and $|e\rangle$ are states of the atom and $|n\rangle$ and $\mid n$ $-1\rangle$ refer to the second laser pulse. Decoherence between these two states will lead to observable effects. The length scale on which one can probe energy decoherence is thus given by the separation between the atom and the light during the waiting period. In the simplest case, the light will still be inside the laser cavity during this time, and will be switched out of the cavity at the right moment. The important distance is then that between the laser and the atom. This distance can be made very large in principle. For example, it is conceivable to connect the laser to the atom by an optical fiber which, depending on the wavelength of the light, would allow distances of several kilometers or more. The fiber has to be interferometrically stabilized for such an experiment, but this seems feasible through constant monitoring of a reference beam. Alternatively, one could consider the use of large free-space interferometers such as those planned for gravitational wave detection [16], which aim for similar distances. Much longer distances could in principle be accessible with space-based experiments. LISA [17] is a project for a space interferometer for the detection of gravitational waves. The interferometer is basically of the Michelson type, with the beam splitter and the mirrors located on satellites separated by $5 \times 10^{9} \mathrm{~m}$. The possible waiting time for the Ramsey experiment is limited by the laser coherence time, which can currently be of order $1 \mathrm{~s}$ [15], corresponding to a distance of $3 \times 10^{8} \mathrm{~m}$. As discussed in section the use of very long-lived metastable states in such experiments should allow one to detect a discretization of time at the level of $10^{-33} \mathrm{~s}$.

\section{Multiatom entanglement}

The sensitivity of Ramsey type experiments could be significantly improved by replacing the single atom with multiatom entangled states of the GHZ type. There have been several proposals for the creation of atomic GHZ states $[14,18,19]$. The recent scheme of Ref. [19] allows the fast creation of approximate GHZ type states, i.e., good approximations to the state $|e\rangle^{\otimes N}+|g\rangle^{\otimes N}$, for large numbers of atoms $N$. The created states are superpositions of two components centered around very different energies. The difference in energy between the two components is of order $N \Delta E$, where $N$ is the number of atoms and $\Delta E$ is the difference in energy between the states $|g\rangle$ and $|e\rangle$ of a single atom. This implies that the gravitational decoherence rate in such a state will be enhanced by a factor of $N^{2}$ compared to a single-atom superposition.

In a generalized Ramsey type experiment one would first create the large superposition state by letting the atoms interact for a certain time in the presence of a laser beam in resonance with the relevant transition [19], followed by a waiting period during which the decoherence could act on the state. Then the laser beam and the interaction would be turned on again (cf. below), leading to a partial revival, whose amplitude would allow one to deduce the amount of decoherence. There are several other decoherence processes in such a scheme whose effects would have to be distinguished experimentally from the quantum gravitational decoherence, in particular atom losses due to spontaneous emission and to inelastic collisions. The loss of a single particle destroys the GHZ type superposition state. The presence of these processes determines the in principle achievable sensitivity for energy decoherence. Discrimination of the different decoherence processes is facilitated by the fact that they scale differently with the particle number and the volume of the system. However, for simplicity and safety we will here assume that the gravitational decoherence rate has to be larger than all other decoherence rates in order for a clear experimental detection to be possible.

We will here discuss the example of strontium, with the atomic ground state ${ }^{1} S_{0}$ as $|g\rangle$ and the metastable ${ }^{3} P_{0}$ state as $|e\rangle$. The advantage of ${ }^{3} P_{0}$ compared to ${ }^{3} P_{2}$ is that inelastic two-body collisions should be strongly suppressed because the inelastic loss channels studied in [20] are absent for the singlet states. It should be possible to create a strontium BEC in either ${ }^{3} P_{0}$ or ${ }^{1} S_{0}$ by optical cooling [21], and also to trap both states simultaneously, as required for the present experiment, using far off-resonant optical traps. The scheme for creating GHZ type superposition states is described in detail in Ref. [19]. What is important for us here is the time scale on which the superposition is created, which is of order $1 /(N \chi)$, where $N$ is the number of atoms in the BEC and

$$
\chi=\frac{2 \pi \hbar}{m V}\left(a_{g g}+a_{e e}-2 a_{e g}\right) .
$$

Here $m$ is the mass of an atom, $V$ is the volume of the BEC and the $a_{i j}$ are the elastic scattering lengths for collisions between two atoms in $|g\rangle$, between two atoms in $|e\rangle$, and between one atom in $|e\rangle$ and one in $|g\rangle$, respectively. For later convenience we define the coefficient $\kappa=2 \pi \hbar / m\left(a_{g g}+a_{e e}\right.$ $\left.-2 a_{e g}\right)$, such that $\chi=\kappa / V$. After creating the macroscopic superposition state, the laser coupling the ground and excited states is turned off, and the parameter $\chi$ is tuned to $\chi=0$, freezing the dynamics. This can be achieved by changing the relative magnitudes of the scattering lengths using, e.g., an optical Feshbach resonance [22]. After a variable waiting period, the laser beam and $\chi$ can be turned on again to induce a revival as detailed in Ref. [19].

For the experiment to be feasible, the time for creating the superposition has to be shorter than all relevant decoherence times. Otherwise decoherence during the creation process would prevent the superposition state from being formed. Moreover, the decoherence rate due to quantum gravity should be comparable to the decoherence due to particle loss. As stated above, we will here assume that it has to be larger. The relevant losses are due to spontaneous emission and to inelastic three-body collisions. The loss rate from spontaneous emission is given by $N \Gamma$, where $\Gamma$ is the spontaneous 
decay rate of the metastable state. The three-body loss rate is of the form $k_{3} N^{3} / V^{2}$, where $k_{3}$ is the three-body loss coefficient. Finally the quantum gravitational decoherence rate that we want to detect is of the form $\gamma N^{2}$, where we have defined $\gamma=\sigma(\Delta E / \hbar)^{2}$.

The requirement that the creation of the superposition has to be faster than the gravitational decoherence gives $\kappa /(N V)>\gamma$, while the requirement that the gravitational decoherence should dominate the other decoherence processes gives the conditions $\gamma>\Gamma / N$ and $\gamma>k_{3} N / V^{2}$. Combining the first and second of these three inequalities gives $V<\kappa / \Gamma$. One should choose $V$ not much smaller than this limit, in order to keep the three-body losses as small as possible. The detectable level of gravitational decoherence is then determined by the two conditions $\gamma>\Gamma / N$ and $\gamma>k_{3} \Gamma^{2} N / \kappa^{2}$, where the first bound varies as $1 / N$ and the second one as $N$. This implies that the smallest possible value for $\gamma$ is attained for $N$ of order $\kappa / \sqrt{k_{3} \Gamma}$. The minimum detectable $\gamma$ is then of order $\sqrt{\Gamma^{3} k_{3}} / \kappa$.

The values of the above quantities can be estimated in the following way. The spontaneous decay rate $\Gamma$ is of order $10^{-3} / \mathrm{s}$ for the extremely long-lived ${ }^{3} P_{0}$ state [23], and the energy separation $\Delta E$ is of order $1 \mathrm{eV}$. The precise values of scattering lengths and inelastic collision rates for $\mathrm{Sr}$ are unknown to the best of our knowledge. However, based on experiments [24] and theoretical calculations [25] for other atomic species one can obtain order of magnitude estimates of $k_{3}=10^{-41} \mathrm{~m}^{6} / \mathrm{s}$ and $\kappa=10^{-17} \mathrm{~m}^{3} / \mathrm{s}$. For these values one finds that the optimal $N$ and $V$ are $N \approx 10^{5}$ and $V$ $\approx 10^{-14} \mathrm{~m}^{3}$, respectively, which is very realistic from an experimental point of view. The minimum detectable $\gamma$ is then approximately $10^{-8} / \mathrm{s}$. This corresponds to a detectable discretization time scale $\sigma$ of order $10^{-38}$ s, five orders of magnitude smaller than what is possible with a single atom. Let us note that naively this time scale corresponds to an ultrahigh energy scale of $10^{23} \mathrm{eV}$. These are only order of magnitude estimates, but it is clear that the use of multiatom entangled states promises a dramatic improvement in sensitivity.

The multiatom states could be integrated into a longdistance Ramsey type experiment. The achievable distance is limited by the decoherence rate. This leads to a tradeoff between sensitivity and accessible length scale. More quantita- tively, the minimum detectable $\gamma$ is of order $\Gamma / N$ (for the above optimal $N$ ), where $\Gamma$ is the spontaneous emission rate, while the decoherence rate is $\gamma N^{2}$, which is of order $\Gamma N$, giving a length scale of $L_{\max }=c /(\Gamma N)$. One therefore has the relation $L_{\max }=\left(c / \Gamma^{2}\right) \gamma$. Putting in the above values, one sees that sensitivity to the minimum $\gamma$ obtained above of order $10^{-8} / \mathrm{s}$ (and thus to $\sigma$ of order $10^{-38} \mathrm{~s}$ ) could still be achieved in an experiment spanning thousands of kilometers.

\section{CONCLUSIONS}

We have argued that global energy decoherence is unobservable in quantum optics experiments, because in practice energy coherence and decoherence are studied by interferometry, which always relies on the use of another system that serves as phase reference. The fundamentally quantum mechanical character of this phase reference is essential for our argument. The observable effects are governed by the relative phase between these two systems, and are unchanged by energy decoherence that acts globally on system and phase reference together. We have suggested that the basic reason for the need for a a phase reference (and thus for the unobservability of global energy decoherence) is the fact that macroscopic objects have very little energy coherence.

However, we have also shown how local energy decoherence, which would act separately on system and phase reference, could in principle be detected with remarkable sensitivity and over a wide range of length scales, from micrometers to millions of kilometers, combining methods from optics and atomic physics. Energy decoherence acting locally below the micrometer scale is already ruled out by atom interferometry. We hope that our present work will provide a motivation for theoretical investigations into the possible existence of a length scale in the predicted quantum gravitational energy decoherence.

\section{ACKNOWLEDGMENTS}

We thank Jorge Pullin for useful discussions, and Todd Brun and Philip Pearle for helpful comments. This work was supported by the IRC network on Quantum Information Processing.
[1] T. Jacobson, S. Liberati, and D. Mattingly, Nature (London) 424, 1019 (2003); G. Amelino-Camelia, J. Ellis, N.E. Mavromatos, D.V. Nanopoulos, and S. Sarkar, ibid. 393, 763 (1998).

[2] J. Ellis, J.S. Hagelin, D.V. Nanopoulos, and M. Srednicki, Nucl. Phys. B 241, 381 (1984); S. Hawking, Commun. Math. Phys. 87, 395 (1982).

[3] R. Penrose, in Mathematical Physics 2000, edited by A. Fokas et al. (Imperial College, London, 2000). The most promising approach for testing this type of predicted decoherence is the creation of superposition states of massive objects in distinct locations, see W. Marshall, C. Simon, R. Penrose, and D. Bouwmeester, Phys. Rev. Lett. 91, 130401 (2003). The decoher- ence rates predicted by the Penrose model for energy superpositions of the kind discussed in the present paper are vanishingly small.

[4] R. Gambini, R.A. Porto, and J. Pullin, Class. Quantum Grav. 21, L51 (2004).

[5] G.J. Milburn, Phys. Rev. A 44, 5401 (1991).

[6] M. Yasuda and H. Katori, Phys. Rev. Lett. 92, 153004 (2004).

[7] J. McKeever, J.R. Buck, A.D. Boozer, and H.J. Kimble, e-print quant-ph/0403121.

[8] J. Finkelstein, comment on Ref. [5], Phys. Rev. A 47, 2412 (1993); see also the reply by G.J. Milburn, ibid. 47, 2415 (1993). 
[9] D. Bedford and D. Wang, Nuovo Cimento Soc. Ital. Fis., B 26, 313 (1975); 37, 55 (1977); I.C. Percival, Proc. R. Soc. London, Ser. A 451, 503 (1995); L.P. Hughston, ibid. 452, 953 (1996); S.L. Adler and L.P. Horwitz, J. Math. Phys. 41, 2485 (2000); S.L. Adler, D.C. Brody, T.A. Brun, and L.P. Hughston, J. Phys. A 34, 8795 (2001); S.L. Adler and T.A. Brun, ibid. 34, 4797 (2001); S.L. Adler, ibid. 35, 841 (2002); Phys. Rev. D 67, 025007 (2003).

[10] R. Bonifacio, Nuovo Cimento Soc. Ital. Fis., B 114, 473 (1999); R. Bonifacio, S. Olivares, P. Tombesi, and D. Vitali, Phys. Rev. A 61, 053802 (2000).

[11] P. Pearle, Phys. Rev. A 69, 042106 (2004).

[12] D.A. Kokorowski, A.D. Cronin, T.D. Roberts, and D.E. Pritchard, Phys. Rev. Lett. 86, 2191 (2001).

[13] L. Hackermüller, K. Hornberger, B. Brezger, A. Zeilinger, and M. Arndt, Nature (London) 427, 711 (2004).

[14] U. Dorner, P. Fedichev, D. Jaksch, M. Lewenstein, and P. Zoller, Phys. Rev. Lett. 91, 073601 (2003).

[15] B.C. Young, F.C. Cruz, W.M. Itano, and J.C. Bergquist, Phys. Rev. Lett. 82, 3799 (1999).

[16] See http://www.ligo.caltech.edu, http://www.virgo.infn.it, and http://www.geo600.uni-hannover.de.

[17] See http://lisa.jpl.nasa.gov and http://www.srl.caltech.edu/lisa/ pubs.html.

[18] J.I. Cirac, M. Lewenstein, K. Molmer, and P. Zoller, Phys.
Rev. A 57, 1208 (1998); J. Ruostekoski, M.J. Collett, R. Graham, and D.F. Walls, ibid. 57, 511 (1998); D. Gordon and C.M. Savage, ibid. 59, 4623 (1999); K. Molmer and A. Sorensen, Phys. Rev. Lett. 82, 1835 (1999).

[19] A. Micheli, D. Jaksch, J.I. Cirac, and P. Zoller, Phys. Rev. A 67, 013607 (2003).

[20] V. Kokoouline, R. Santra, and C.H. Greene, Phys. Rev. Lett. 90, 253201 (2003).

[21] M.D. Barrett, J.A. Sauer, and M.S. Chapman, Phys. Rev. Lett. 87, 010404 (2001).

[22] H. Feshbach, Ann. Phys. (N.Y.) 5, 357 (1958); S. Inouye, M.R. Andrews, J. Stenger, H.-J. Miesner, D.M. Stamper-Kurn, and W. Ketterle, Nature (London) 392, 151 (1998); T. Calarco, U. Dorner, P. Julienne, C. Williams, and P. Zoller, quant-ph/ 0403197. M. Theis, et al., Phys. Rev. Lett. 93, 123001 (2004).

[23] See M. Takamoto and H. Katori, Phys. Rev. Lett. 91, 223001 (2003); G. Ferrari et al., ibid. 91, 243002 (2003); I. Courtillot et al., Phys. Rev. A 68, 030501 (2003).

[24] E.A. Burt, R.W. Ghrist, C.J. Myatt, M.J. Holland, E.A. Cornell, and C.E. Wieman, Phys. Rev. Lett. 79, 337 (1997); A. Görlitz et al., ibid. 90, 090401 (2003).

[25] E. Tiesinga, S. Kotochigova, and P.S. Julienne, Phys. Rev. A 65, 042722 (2002); B. Bussery-Honvault, J.-M. Launay, and R. Moszynski, ibid. 68, 032718 (2003). 